\title{
CANCER
}

\section{An immunocompromised zebrafish to test patient-derived xenografts}

Yan, C. et al. Cell https://doi.org/10.1016/j.cell.2019.04.004 (2019)

David Langenau at Massachusetts General Hospital Research Institute has long worked with zebrafish to study pediatric cancers. His early work involved transgenic lines, but such animals can only take things so far. "There's really something about seeing the heterogeneity of drug responses in patient tumors that is required to move drugs forward," he says. Patient-derived xenograft (PDX) mouse models have been standard for preclinical drug efficacy testing, but a fishier version has been in the works.

It's worked in zebrafish...larvae. After hatching larvae lack an adaptive immune system, but that immunity eventually kicks in and kicks out foreign cells, limiting PDX studies to about a week. The small fish can only handle a few hundred cells at time, compared to the millions engrafted into a mouse. Larvae cannot be gavaged either; instead, their water is spiked with a therapy, "but you don't really know how much drug an animal is taking up," Langenau says. That water is also a bit cold: larval zebrafish do best below $35^{\circ} \mathrm{C}, 2{ }^{\circ} \mathrm{C}$ lower than the human body.
Writing in Cell, Langenau and colleagues introduce an immunocompromised adult zebrafish to overcome larval limits. They developed a prkdc $^{-/}$, il2rga ${ }^{-/-}$mutant line lacking immune cells that would otherwise fight off the transplant. These adults are also clear; no pigment, or fur, to get in the way of imaging, which can be done using simple confocal microscopy. "The most interesting aspect in the paper is probably the ability of our model to provide single-cell imaging of engrafted cells," says first author Chuan Yan. Solving the genetic component was actually the easy part. "The major hurdles were growing the fish at $37^{\circ} \mathrm{C}$," says Langenau. "That's never been done before." But the fish acclimated, with some feeding tweaks to accommodate their faster metabolisms in the warmer water and some antibiotics to prevent infection.

In the paper, Yan et al. show lasting engraftments of several solid tumors. Compared against murine PDX models, tumor biology in the zebrafish followed similar trajectories, and drugs had similar effects. There's room to improve engraftment efficiencies for different cancers, but Langenau envisions a 'zebrafish avatar army', with multiple therapies tested against a patient-derived tumor in the fish before moving the most promising candidates on.

"I was particularly impressed with the effort to perform pharmacokinetics and match the drug exposure in fish as close as possible to humans. This is an essential aspect of preclinical testing," commented Michael Dyer, who provided xenograft samples through the Childhood Solid Tumor Network at St. Jude's. "I am excited to see how this system expands to other pediatric solid tumors. It is essential to distribute the protocols and procedures to the broader community so that other experts in zebrafish can begin similar studies."

\section{Ellen P. Neff}

Published online: 17 June 2019 https://doi.org/10.1038/s41684-019-0338-2

\section{natureresearch} ACADEMIES

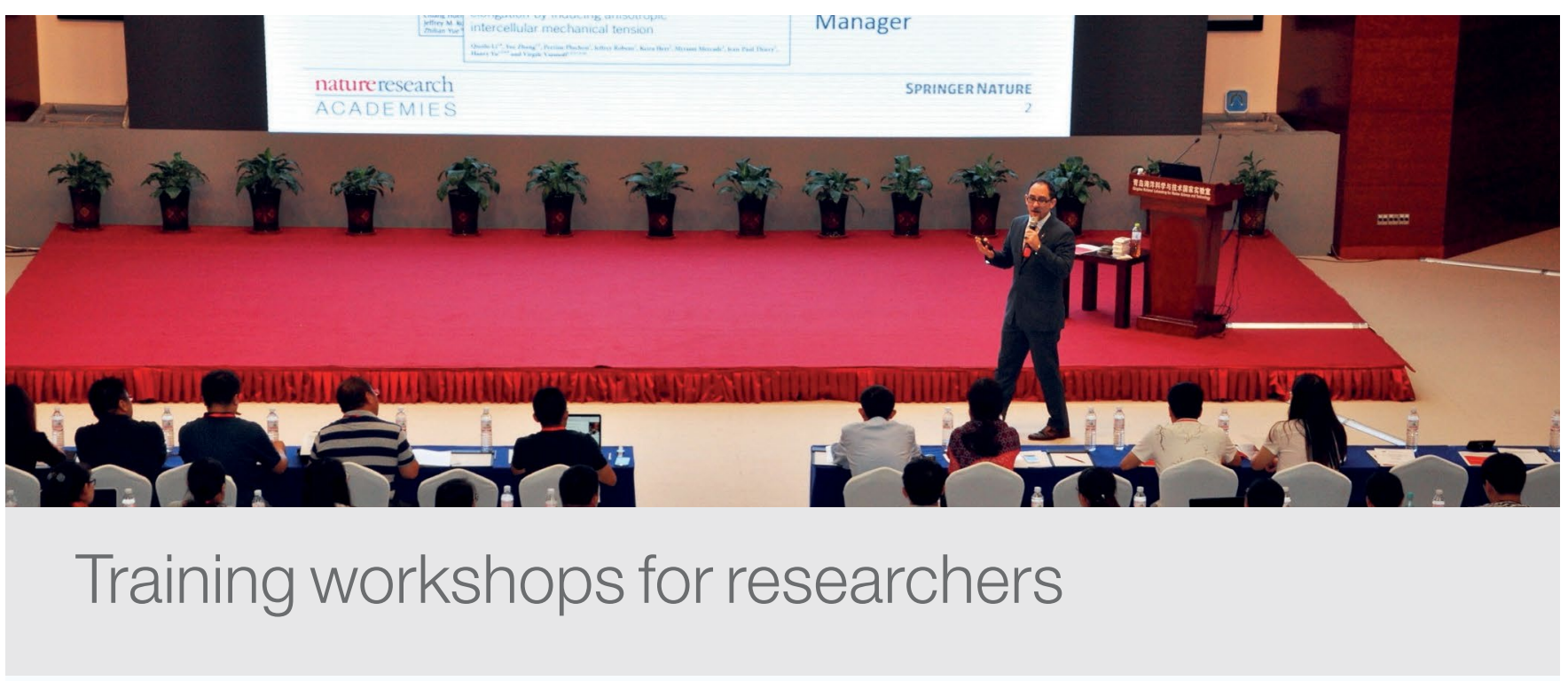

A series of workshops to support researchers, covering topics such as getting published, journal editing, clinical research methodology, and applying for research positions.

Visit partnerships.nature.com/academies to host an academy at your institution. 